\title{
Amplified Music and Sound Level Management: A Discussion of Opportunities and Challenges
}

\author{
JOHANNES MULDER, AES Member \\ (j.mulder@murdoch.edu.au) \\ School of Arts, Murdoch University, Perth, Western Australia
}

\begin{abstract}
Music induced hearing disorders and noise pollution emerge on the crossroads of technology, culture, and society. This paper argues that multidisciplinary approaches are required to reduce hearing risks at music concerts and related problems of noise pollution. The paper segues from a broad to a narrow perspective looking at the interactions between stakeholders "on the floor." The paper argues that even though noise regulations and hearing risk mitigation policies may be different between nations and states, best practices originate from procedures and policies that are developed from an understanding of the multiple stakeholder perspective and that facilitate dialogues between the different agents involved.
\end{abstract}

\section{INTRODUCTION}

At live music concerts many different perspectives on sound pressure level (SPL) come together. This can be exemplified by listing the stakeholders: audiences, musicians, venues, promoters, agents, sound hire companies, sound engineers, hearing risk advocacy, occupational and health safety (OHS) agencies, and, last but not least, the neighbors. A rock band may have the desire to "be" loud from an equally aesthetic as well as experiential ideal; their representative sound engineer at the mixing desk is, or should be an ambassador for those ideas. The system's engineer or host at that mixing desk can be seen as a representative of the venue who are (often) legally bound to adhere to certain limits, either from OHS, environmental regulation or a voluntary limit set by the venue. Audiences, ranging from a few dozen to tens of thousands or more people, have different individual expectations and preferences; some people may bring, but not necessarily wear or insert correctly, earplugs; some may be under the influence of alcohol or other drugs (legal or illegal) subduing or enhancing their experience. Outside of the venue residents or other users of the public space might experience the concert as pollution, as interfering with their soundscape, keeping people from sleeping, or resulting in other detrimental effects associated with uncontrollable sound. In relation to music the matter of the soundscape is ambiguous; generally, or rather historically speaking, music is a common element of our soundscapes, with important cultural and social values and histories. However since the genesis of electronically mediated (and amplified) sound, music has transitioned permanently into both the private sphere (with the ubiquitous headphone as a trump) and, at the same time, into the public sphere, as a consequence of the increased levels.

In this paper I want to tease out the interactions between the perspectives listed above in order to reinterpret the options for the different stakeholders as agents with regard to the issue of sound level management. Combining a discussion of existing literature from a number of academic and scientific disciplines with some of my own experiences as a live sound engineer, I will argue that common issues of live amplification, hearing damage risk, and disturbance of local soundscapes need to be addressed in a single strategy, even though different regulations may be in force. Live concerts play a vital role in the current day cultural economy in particular since revenues are on par or have overtaken the recorded music industry $[1,2]$. Its sustainability needs to be ensured by empowering stakeholders to cooperatively develop approaches to hearing damage risks and noise pollution.

\section{SWEEPING STATEMENTS}

In an article in the Guardian [3] readers are advised to wear earplugs when going to pop concerts. While that is great advice, the responses in the comments section raise some revealing questions. A reader wonders why concerts are so loud and why there appear to be no regulations? And why is the sound crew not constrained to operate within the limits of hearing damage? The suggestion that the problem is simply sound engineers turning up the volume (their motivations are left undiscussed but presumably because they can) can be observed regularly suggesting that the solution is simply telling the sound engineer to turn it down. 
Two more examples come from the popular website The Conversation; a reader in response to an article [4] with the imaginative title "Live Music in Australia: Offensive Noise or Good Vibrations" writes:

I often wonder whether the problem is the sound technicians - they set the volume of the speakers, balance the various tracks etc. [...] how deaf are the sound technicians? Is the music so loud just so they can hear something?

In a discussion on that same site by an acoustician researching hearing risks for (classical) musicians [5] the author writes:

The challenge is actually greater in classical orchestras, which produce unamplified music. In amplified music you can simply turn the volume down, which is one of the tasks of the sound or recording engineer.

This author not only mixes up the task of a recording engineer with that of a live sound engineer he ignores the complexity of multiple sound sources (including sometimes the audience singing and yelling along) coming together in an acoustic space, which may or may not be suitable for amplified music. Unskilled and untrained sound engineers are not uncommon (these are often low-wage jobs or volunteers' roles). A recent survey [6] in the UK reveals the wide range in educational and professional backgrounds among people working in live sound. The study shows an increase in tertiary education providers offering courses in live sound or specialist modules to students studying sound (recording) engineering or music technology. According to the survey many musicians and engineers agree that SPLs are often too high and, even though wearing hearing protection (HP) is important for all involved, efforts to bring down SPLs should be preferred.

\subsection{WHY LOUD?}

Barry Blesser [7, 8] looks at some aspects of loud music, cautioning that it has been well established that exposure to loud sounds causes hearing damage, but the question why people like loud music has been insufficiently researched. One obvious reason for the lack of research is that subjects cannot be asked to submit themselves to very loud music, so studies are usually indirect. Another issue that complicates research is that the context for musical events can be very different, e.g., venue location (indoor/outdoor, small/large), genre (and target audience), or duration (a single headliner to a five-day festival). These entirely different situations require tailored approaches to comply with regulations while assuring audience satisfaction. Blesser [7] argues that excessively loud music serves a purpose and is not:

-An accident that arises from ignorance of the consequences;

-The result of being manipulated for commercial profits;

-A temporary fad that happens to exist in our culture at this moment in time.
Blesser points out that loud sound can be found in many places-at sporting events, in cars, cinemas, and the ubiquitous personal stereo. Gyms [9], with their high-energy group workouts, should be added to that list.

\subsection{The Desire to Be Loud}

Loud music amplification is not limited to pop music. Fluxus composer and minimalist La Monte Young often prescribed loud amplification for performances of his works in the 1960s. According to Keith Potter [10] in his book Four Musical Minimalists, high amplification levels were needed to "explore the innards of sound," meaning harmonic partials, "with full clarity and intensity." Composer Philip Glass, in an interview in SPIN [11], remembers that after hearing Jefferson Airplane play at the Filmore East in Manhattan, he wanted to be like that: "I want to be loud." And, "When repetitive structured music [...] is played loud, you begin to hear kind of the froth-it's like a river that sends up all this white foam. It was the foam I was interested in." In another publication [12], Glass is cited about the same phenomenon: "You actually hear sounds that nobody is playing, a shiny top to the music."

With the amalgamation of jazz and rock led by Miles Davis in the early 1970s, loud amplification reached a broader audience:

When (Ron) Lorman began mixing the sound for Miles's concerts, he faced a dilemma. Half of the audience seemed to comprise older fans that had grown up listening to Sketches of Spain and Kind of Blue, and the rest were younger members keen to hear the louder and more aggressive sound delivered by the new band. Lorman approached Miles and told him about this audio paradox, adding that he was looking for a happy medium. "He looked at me and paused for what seemed like an eternity and said: "Ron. Turn it up—make it loud. I want them to feel it." [13]

Two arguments emphasize that the sound engineer's job is more complex than controlling volume. First, as illustrated by the citation, that sound engineer's decisions can be informed by the wishes of the musicians she or he represents; second, if the sound engineer were to turn down the volume completely a venue would not go silent. An ordinary band with drums and electric guitars can produce very high on-stage SPLs, in (smaller) indoor venues in particular. In such venues, during the soundcheck, often the first point of call for a sound engineer in terms of bringing sound levels down is the performers on stage, asking them to turn down guitar amplifiers and other backline, or aiming them away from the audience. Musicians don't necessarily comply with such requests; sometimes going back to the pragmatic, or even ideological argument that music has to be loud because their music (genre) is loud music [14]. Guitarists often argue that in order to create their "sound" an amplifier needs to be at maximum level (not seldom a reason for performers to play wearing HP). In general, as a sound engineer it would not be appropriate to, for instance, ask a band to send the drummer home, or tell the drummer to play on trigger pads (playing back samples) instead of 
an actual drum kit. Some acts and their audiences cultivate extreme sound levels, e.g., the drone band Sunn O))), the name and the parentheses refer to the former powerful guitar amp brand [15].

\subsection{Practical Reasons}

Apart from artistic, personal preference, and cultural reasons why some stakeholders like their concerts loud, a few practical and technical reasons can be listed.

\subsubsection{Overcoming the Audience}

Overcoming a screaming (singing along) crowd (e.g., unheard concerts by The Beatles or relentless screaming at appearances of popular bands such as One Direction). Loud amplification allows each member of the audience to sing or yell along while at the same time experience the performance on stage.

\subsubsection{Overcoming the Band}

For a mix engineer to have full control over a mix, over what the audience hears, the backline and monitor needs to be drowned out in order to work independently from the stage sound. Musical instruments of choice in some genres are loud to begin with, a drum kit played by a strong, rocking drummer with the thickest drum sticks; a guitarist who achieves his preferred sound by maximizing the volume of his amplifier. Elsewhere, in a chapter about iconic transducers [16] I have written about the importance of the visibility of guitar amplifiers (e.g., large Marshall stacks) on stage. In some cases this relegates amps to stage props with the actual amplifier in use hidden off-stage, not adding to the onstage sound level. Although large numbers of performers use in-ear monitors when performing (that certainly helps in reducing on-stage sound levels, and can aid in abating hearing damage risks [17]) common monitor loudspeakers (aimed at the performers, away from the audience) add to the sound level. In smaller venues stage monitors can "leak" low and low-mid frequency energy towards the audience, which has to be overcome by an increase in sound level from the PA system.

\section{LOUDNESS}

Loudness is a perceived, subjective quality and empirical data can only be gathered by asking people about it. Like pitch it is a psychological phenomenon. Daniel Levitin [18] (p. 67) writes: "The point here is that it takes a brain to experience what we call 'loudness.",

He goes on to discuss concertgoers, some of whom like very loud or extremely loud music. He mentions reports of "a special state of consciousness, a sense of thrills and excitement, when the music is really loud."

Models such as the K-weighted Loudness Unit can aid in predicting measurements. Recent work by Simon Durbridge et al. [19] looks at the relation between different types of distortion and loudness perception in a level setting experiment. Although more work is required the suggestion is that specific distortion added in the digital domain can increase the loudness experience.

\subsection{Motivations}

Blesser [7] suggests three separate but related motivations why some people like loud music: "social rewards, biological stimulation, and selective aural focus." The latter aims at the drowning out effect of the loudest sound, "loud sound dominates the aural space." Biological stimulation, Blesser suggests, like Levitin, a special (altered) state of consciousness; about which he remarks that it unjustly suggests there is a "normal" state [20]. Finally Blesser signals a relation between loud music and social cohesion: "Loud sonic events are bigger, closer, and energetic" and "A live concert, even with reproduced music and lipsyncing performers, provides a connection to others in the audience." [21]

\subsection{Physiological Aspects}

Research in this area is limited by the impossibility of subjecting subjects to dangerously loud sound. One notable exception is found in the work by Todd and Cody [22]. With careful monitoring of subjects' safety the researchers managed to measure vestibular system responses up to 120 $\mathrm{dB}_{(\mathrm{A})}$. The vestibular system, including the saccule, is responsible for our bodily balance; it shares the labyrinth of the inner ear with the cochlea of our hearing system. The authors anecdotally link this vestibular response to "sensations of self-motion" such as experienced on a swing (which is, similar to loud music, something that some people like more than others). The vestibular response appears to occur at levels above an individual threshold ranging from 90-120 $\mathrm{dB}_{(\mathrm{A})}$ (in the case of Todd and Cody's work the range of the stimuli). In the conclusion the paper suggests that there could be a relation between the findings and what is referred to as the "Rock and Roll Threshold" that was proposed by Ken Dibble [23]. Christian Lehman [15], referring to Todd and Cody's work writes that this explains why we cover our ears at loud screeching machine noise, but "voluntarily listen to and enjoy loud disco or techno music with booming bass." A more recent paper [16] refers to Todd and Cody's hypothesis, and Dibble's threshold is still mentioned as a possibility:

The vestibular hearing lies in the range of aloud low frequencies (50-800 $\mathrm{Hz}$ and above $90 \mathrm{~dB}_{\text {spl }}$ ), which may be obtainable from loud dance music or overt singing. This response can be a physiological basis for the minimum loudness necessary for rock and dance music, so, vestibular hearing contributes to the affective quality of loud sounds.

In a recent study of the Iranian daf (a loud percussion instrument) [24]:

The intensities and frequency distributions that are typical in percussive music, such as rock concerts, dance clubs and celebrations, seem almost designed to stimulate the saccule. 
The minimum level at which this vestibular response is different for each subject (i.e., the individual threshold that Todd and Cody describe); for some it starts at $90 \mathrm{~dB}_{\text {spl }}$ for other at $120 \mathrm{~dB}_{\text {spl }}$, at the threshold of pain. This might be an explanation for the general subjectivity of the live music experience and common disagreement of what constitutes a comfortable sound level.

\subsection{Music as Nuisance}

"Unfortunately, our culture has linked loudness with enjoyment," writes Arline Bronzaft (quoted in [25]). Music as nuisance predates contemporary sound technology, as witnessed by Immanuel Kant [26]:

Over and above all this, music has a certain lack of urbanity about it. For owing chiefly to the character of its instruments, it scatters its influence abroad to an uncalledfor extent (through the neighborhood), and thus, as it were, becomes obtrusive and deprives others, outside the musical circle, of their freedom.

Noise pollution as both a general health and a mental health issue has been well established [27, 28]. Consequentially, regulations in many developed countries have labeled the unwanted, second hand, by-products of amplified concerts as noise (which is not the same as equating music to noise). In some countries (Australia for instance) music is susceptible to a $\mathrm{dB}$ penalty in comparison to other noise sources, as a consequence of its "tonal, modulating or impulse characteristics. [...] low frequency, modulating or tonal dominated noise is more annoying than a constant, broad and steady noise" [29]. This effectively adds $10 \mathrm{~dB}$ or $15 \mathrm{~dB}$ to environmental measurements, the higher number is for sounds with "impulsive bass beats," a lower, $5 \mathrm{~dB}$ penalty is applied to sounds with just a tonal component, e.g., HVACs.

\subsection{Stakeholders}

Kim Kähäri [30] is coauthor of a paper discussing an intervention at a small Swedish music venue in order to reduce the average sound levels. Point of departure was the question whether this could be done without the "compromising artistic freedom" to "perform at an intended sound level," or the patron's "listening experience." A report from Preventing Hearing Loss (AES Tutorial 120th Convention, 2006) $[31,32]$ mentions work done by Kahari, looking at creating a healthy live-sound environment with "quality sound at reduced levels." One recommendation suggests setting up meetings with stakeholders, "including the musicians' union, sound technicians, university staff, event organizers, and health professionals." Such stakeholder gatherings are a good way of asserting that this is not just a problem of sound engineering or music culture.

A relevant example comes from the Netherlands where the successful "hearing covenant" [33] between concert production stakeholders, hearing protection advocacy NGO, and the national government was renewed for three more years. That covenant prescribes a 15 minute Leq $=$ $103 \mathrm{~dB}_{(\mathrm{A})}$ replacing short term measurements (e.g., one minute and fast), peak value is set at $140 \mathrm{~dB}_{(\mathrm{C})}$. Other re- cent examples come from Belgium and Switzerland where similar dosage-based regulation can be found with values of $100 \mathrm{~dB}_{(\mathrm{A})}$ over one hour. In Germany it was made a DIN-norm with a maximum of $99 \mathrm{~dB}_{(\mathrm{A})}$ over two hours with an extra headroom of $102 \mathrm{~dB}_{(\mathrm{A})}$ for 30 minutes [34]. An important element of these new approaches is that SPL levels must be logged and archived, which is an important step for both research and regulation in this area.

\section{SOUND LEVEL MANAGEMENT}

From this broad perspective I want to zoom in on the interaction between stakeholders and their agents before and during a concert. As anecdotally established at the beginning of this paper, the technicalities of the issue at hand are at risk of being simplified to an amplified versus non-amplified binary, with a volume control in some central place; all the sound engineer does is turn the volume up (or down).

\subsection{Signal Chain with Agents}

Following the chain of events, actors and agents causing or triggering musical sounds in some of the interrelated complexities can be drawn out. A band, playing guitars, banging on drums, and singing is the source that utilizes different transduction and conversion technologies to feed the amplification system, The instruments and individual instrument amplifiers (referred to as backline) create a reference sound source for the performers, often enhanced by (in-ear) monitors playing back specific mixes for each performer. Backline and monitor loudspeakers are essential on-stage sound sources that not always interact favorably with the PA addressing the audience. The same signals feeding the monitor mix are balanced by a dedicated front of house $(\mathrm{FOH})$ mix engineer (generally a representative of the performers) and sent to the amplifiers and loudspeakers. The sound system is usually optimized and hosted by a systems engineer, a representative often of both the provider of the sound equipment (sound hire) and by proxy the venue and or event promoter. Mix engineer and system engineer, often supported by a "Sound Guard" [34] form a sound level management firm (often an acoustics consultant utilizing calibrated specific sound level measurement tools) or a stand-alone set of tools (ranging from a mobile phone app to dedicated software running on a computer with calibrated hardware).

\subsection{Agency and Sound Levels}

During a concert these three agents have to optimize the outcomes for a number of stakeholders:

1) The band (who may insist on high SPLs as a consequence of the genre they identify with);

2) The audience who may like a concert to be loud to enhance their experience; who may or may not be protecting their hearing with ear plugs or anything in between. Occasionally audience members will address the sound engineers at the mixing desk (sometimes 
with a sound level meter phone-app at hand) to tell the mixer to turn the level up or down. This illustrates again how many opinions and directions need to be kept in balance;

3) The venue/promoter who wants, simultaneously, to assure an optimized experience for their audiences but also wants to be sure to stay within regulatory limits. Infringements may result in fines or perhaps a loss of license, which can be a valuable asset;

4) People in the urban area surrounding the venue can sometimes be the largest stakeholder group. And unless a hotline for complaints is provided they can only complain after the event, after the damage is done. At outdoor events this can be a very large area, depending not just on levels but also system design and meteorological circumstance. An example of an appeasing strategy is inviting the neighbors to the event providing them with VIP treatment and so increasing the stake by inviting them to become part of the audience.

\subsection{Inner City Venues}

In the outdoors sound roams free, inside a venue acoustic conditions can significantly influence the SPL of a concert. Not just from the PA addressing the audience but initially also from on-stage instruments, backline, and floor monitors. For many bands there is no difference in set-up and requirements whether on a large festival stage, a dedicated pop venue or in a small band room where the audience is practically on stage with the musicians and their amps. Many smaller venues (or even pubs and hotels) are acoustically inadequate and lack the funding for much needed acoustic improvement or even an up to date PA system. The inner-city location of such venues adds to the challenge of reducing sound levels to protect patrons' ears, which may very well require a different set of metrics in comparison to outdoor and larger indoor venues.

\section{DOSAGE BASED MEASUREMENT}

As a consequence of the great range in duration, concerts are hard to express in the usual $3 \mathrm{~dB}$ exchange rate, except for venue staff working a known number of hours in potentially loud environments where employers have to work with OHS regulation.

Sound level measurement software packages such as 10Eazy [35] are designed to better inform the agents. Running on dedicated laptops, with calibrated audio hardware these tools are used at the FOH mixing desk, which is also the position of the measurement microphone. In combination with dosage-based maxima (like the European examples mentioned before) the interface alerts the agents to a possible exceedance within the specified time frame and suggests a correction. Different to traditional SPL-meters, the information provided by the software facilitates a dialogue between mixer and system engineer (and/or sound guard), which replaces the potentially conflict-inducing instruction: "turn it down, now!" Research [36] undertaken at a large festival using measurements from dosage meters

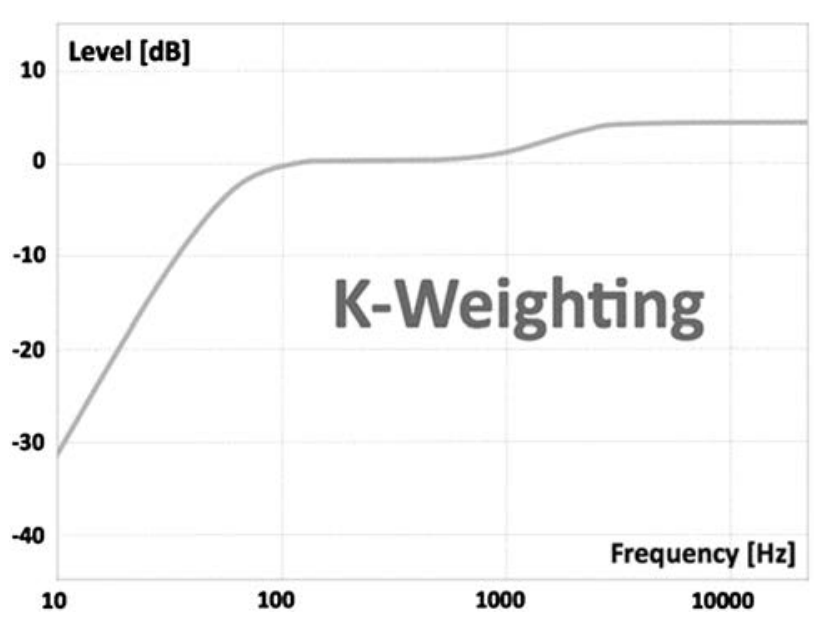

Figure 1 "K-Weighting" filter curve for loudness measurement from EBU-TECH 3343

worn by audience members, suggests a good correlation between $\mathrm{FOH}$ based measurements (often 20 meters or more away from the stage) and the levels experienced by the audience.

\subsection{Dynamic Range: An Opportunity}

The significant opportunity that emerges is that technology facilitates a dialogue that supports the mixer to work dynamically, instead of trying to maximize to an instantaneous $\mathrm{dB}$ value. Extending the dynamic range of a concert will allow for a reduction in average loudness and exposure for the audience, potentially also reducing emission into the neighborhood. This scenario could establish a practice that prepares the dynamic range of a show beforehand, informed by a band's set list. Potentially this process takes place in discussion with the musicians, which will ultimately make them a stakeholder, not only of their "live" dynamic range but also of the wider debate of live music and desired SPL.

The importance of dynamic range has been very well demonstrated by the work done for ITU BS.1770 and the EBU-R128 recommendation in terms of loudness normalization for broadcast audio [37]. Trading-in peak level maxima for loudness-based measurements (using Loudness Units and the $\mathrm{K}$-Weighting) is proving to be a peace process in the loudness war. The current practice of prescribing instantaneous or short term maxima in combination with peak limiters (very common in many fixed sound installations), similar to broadcast audio, reduce dynamic range, and increase the exposure of an audience.

Venues and festivals, in coordination with other stakeholders can implement dosage based SPL maxima that are considered safe and appropriate for venue size, acoustics, genre, and other relevant factors. With or without official regulations such metrics can be seen as a tool that helps shape or compose the dynamic range of a concert.

\subsection{Dedicated Weightings for Live Sound?}

Loudness Units and the K-weighting, with its reduced LF response, are of limited use for live sound unless in 
combination with the C-curve. But its successful implementation does raise the question whether the common Aweighting, even in combination with a $\mathrm{C}$-weighted value, is still the best way to go for sound level management.

The question of the appropriateness of the A-weighting for sound level management has been raised before [31, 38, 39]. The issues are well-known-the A-weighting is based on the equal loudness curve at 40 Phon, established using pure tones and has a strongly reduced LF weighting. This reduced weighting of low frequencies in the A-curve makes it inappropriate for measurement of environmental impact, which is much stronger in LF. Consequentially, local environmental regulations and event permits usually specify a separate level for A and C. In the light of sound level management this issue was discussed at a panel session at the $58^{\text {th }}$ AES Conference. The panel concluded that at this moment in time a change of the trusty $\mathrm{dB}(\mathrm{A})$ is unlikely for the simple reason that every (SPL-) meter and presumably every piece of sound level regulation is informed by it. Other challenges mentioned for an up-to-date weighting are large differences in spectral content between genres (e.g., a rock, country or dub step); the (spectral) difference between measurements inside and outside a venue, and the big difference between large and small venues where the SPL is driven directly by the backline.

\section{CONCLUSION}

The creative economy of a city requires a functioning live music industry, while at the same time a sustainable city with a high quality of life has increasing levels of control over its soundscape, whether looking at noise pollution or helping people to protect their hearing. As I argue in this paper this requires new, better informed, innovative approaches to amplified music that combine top-notch technologies with good understanding of stakeholder relations and needs. The different perspectives outlined in this paper and more particularly the complexity that emerges around the interaction of stakeholders before and during concerts, urge for interdisciplinary and innovative approaches in terms of policy development, education (of musicians and sound engineers), and research. The challenge for the stakeholders identified in this paper lies in finding best practices that address both issues of noise pollution and hearing risk mitigation. Such challenges are different depending on venue size, music genre, and acoustics. However, regardless of these parameters the stakeholders that can be identified are similar in most situations, so lessons learned and best practices can be shared and adapted or further developed to fit different situations.

With loudness normalization in broadcast audio as inspiration there is an opportunity for the live sound industry. Dosage approaches to sound level management, whether through regulation or self-imposed, offer the opportunity to increase dynamic range and reduce loudness exposure. At the same time, novel software interfaces can take the interactions between stakeholders from one of policing to one of empowerment.

\section{ACKNOWLEDGMENTS}

I would like to thank the reviewers of this article and the panel on Sound Level Management for Musical Events at the $58^{\text {th }}$ AES conference in Aalborg 2015: Michael Santucci (Sensaphonics, USA), Thomas Lund (TC Electronics, DK), Marcel Kok (dB Control, NL), and Jacob Navne (10eazy, DK). The latter two have contributed greatly to my research efforts on this topic, for which I am very grateful. Also I would like to thank Esben Skovenborg (TC Electronics) for his contributions to the discussion. Part of this work was supported by CAESIE grant no. 1657388 . This article was originally presented at the $58^{\text {th }}$ AES conference on Music Induced Hearing Disorders [40]. Some of the literature reviewed in this paper was first described in [41]. Finally I would like to thank Simon Durbridge (University of Derby) and John Taylor (D\&B Audio) for sharing their work.

\section{REFERENCES}

[1] F. Holt, "The Economy of Live Music in the Digital Age," European J. Cultural Studies, vol. 13, no. 2, pp. 243 261 (2010). http://dx.doi.org/10.1177/1367549409352277

[2] M. Connolly and A. B. Krueger, "Rockonomics: The Economics of Popular Music," NBER Working Paper No. 11282 (2005).

[3] L. Diluter "Should I Wear Earplugs to Concerts?," The Guardian (2012) [Accessed 23 December 2015]; Available from: http://www.guardian.co.uk/lifeandstyle/ 2012/may/13/should-wear-earplugs-to-concerts.

[4] A.C. North "Live Music in Australia: Offensive Noise or Good Vibrations?," The Conversation (2013) [Accessed 10 March 2015]; Available from: http://theconversation.com/live-music-in-australiaoffensive-noise-or-good-vibrations-13530.

[5] S. Dance "Musicians at High Risk of Hearing Loss, but the Show Can Go On," The Conversation (2014) [Accessed 10 March 2015]; Available from: https://theconversation.com/musicians-at-high-riskof-hearing-loss-but-the-show-can-go-on-26134.

[6] A. Jamieson "Use of and Attitudes Towards Hearing Protection in the Sound and Music Industries," presented at the AES 58th International Conference: Music Induced Hearing Disorders (2015 June), conference paper 1-2.

[7] B. Blesser, "The Seductive (Yet Destructive) Appeal of Loud Music," Econtact, Communauté électroacoustique canadienne / Canadian Electroacoustic Community (2007).

[8] B. Blesser and L.-R. Salter, "The Unexamined Rewards for Excessive Loudness," Communications: 9th International Congress on Noise as a Public Health Problem (ICBEN) (2008), Foxwoods, CT.

[9] E. F. Beach and V. Nie, "Noise Levels in Fitness Classes Are Still Too High: Evidence from $1997-$ 1998 and 2009-2011," Archives of Environmental \& Occupational Health, vol. 69, no. 4, pp. 223-230 (2014). http://dx.doi.org/10.1080/19338244.2013.771248

[10] K. Potter, Four Musical Minimalists: La Monte Young, Terry Riley, Steve Reich, Philip Glass (Cambridge University Press, Cambridge, 2002). 
[11] W. Hermes, "Glass' Menagerie," in SPIN2008, pp. 96-100 (2008).

[12] A. Kozinn, "The Touring Composer as Keyboardist (1980)," in Writings on Glass: Essays, Interviews, Criticism, R. Kostelanetz and R. Flemming, Eds. (University of California Press, Berkeley, 1999).

[13] G. Cole, The Last Miles: The Music of Miles Davis, 1980-1991 (The University of Michigan Press, Ann Arbor, MI, 2005), p. 534.

[14] J. M. Parcival, "Stone Deaf Forever: Discourses of Loudness," Volume!, vol. 11, no. 2, p. 20 (2015).

[15] O. Lucas, "Maximum Volume Yields Maximum Results," J. Sonic Studies, vol. 1, no. 7 (2014).

[16] J. Mulder, "The Iconic Microphone," Academic Quarter, vol. 10 (Spring 2015).

[17] H. Malyuk, R. Schulein, and M. Santucci "New Developments in In-Ear Monitor (IEM) Technology for Musicians with Hearing Loss," presented at the AES 58th International Conference: Music Induced Hearing Disorders (2015 June), conference paper 5-1.

[18] D. J. Levitin, This Is Your Brain on Music: The Science of a Human Obsession (Plume, San Francisco, 2007).

[19] S. E. Durbridge, A. J. Hill, and J. Taylor, "The Effects of Distortion on the Perception of Loudness in Live Sound," Proc. Institute of Acoustics Conference on Reproduced Sound, vol. 37, pt. 4 (2015).

[20] G. Gerra, et al., "Neuroendocrine Responses of Healthy Volunteers to 'Techno-Music': Relationships with Personality Traits and Emotional State," International J. Psychophysiology,vol. 28, no. 1, pp. 99-111 (1998). http://dx.doi.org/10.1016/S0167-8760(97)00071-8

[21] B. Tarr, et al., "Synchrony and Exertion During Dance Independently Raise Pain Threshold and Encourage Social Bonding," Biology Letters, vol. 11, no. 10 (2015). http://dx.doi.org/10.1098/rsbl.2015.0767

[22] N. Todd and F. Cody, "Vestibular Responses to Loud Dance Music: A Physiological Basis of the 'Rock and Roll Threshold," J. Acoust. Soc. Am., vol. 107, no. 1, pp. 496-500 (1999). http://dx.doi.org/10.1121/ 1.428317

[23] K. Dibble, "Hearing Loss and Amplified Music," J. Audio Eng. Soc., vol. 43, pp. 251-266 (1995 Apr.).

[24] S. F. Emami, "Acoustic Sensitivity of the Saccule and Daf Music," Iranian J. Otorhinolaryngology, vol. 26, no. 2, serial no. 75, pp. 105-110 (2014).

[25] K. Bijsterveld, Mechanical Sound: Technology, Culture, and Public Problems of Noise in the Twentieth Century (MIT Press, Cambridge, MA 2008).

[26] I. Kant, Critique of Judgement (Clarendon Press, Oxford, 1978).

[27] S. A. Stansfeld and M. P. Matheson, "Noise Pollution: Non-Auditory Effects on Health," British Medical Bulletin, vol. 68, no. 1, pp. 243-257 (2003). http://dx.doi.org/10.1093/bmb/ldg033
[28] D. Halpern, Mental Health and the Built Environment: More than Bricks and Mortar? (Rutledge, 2004).

[29] Environmental Protection Agency OBJ Guidelines for the Use of the Environment Protection (Noise) Policy 2 (2009); Available from: http://www.epa.sa.gov.au/ files/F47715_guidelines_noise_epp.pdf.

[30] J. Sandell, et al., "Acoustic Intervention in a Live Music Club," Acta Acustica United With Acustica, vol. 93, no. 5, pp. 843-849 (2007).

[31] Preventing Hearing Loss, presented at the 120th Convention of the Audio Engineering Society (2006 May), Tutorial 7.

[32] K. Kähäri, et al., "Assessment of Hearing and Hearing Disorders in Rock/Jazz Musicians: Evaluación de la audición y de los problemas auditivos en músicos de rock y jazz," International J. Audiology, vol. 42, no. 5, pp. 279-288 (2003). http://dx.doi.org/10.3109/ 14992020309078347

[33] M. Van Rijn "Convenant preventie gehoorschade muzieksector)" (2015) [Accessed 20 December 2015]; Available from: https://www.rijksoverheid.nl/documenten/ kamerstukken/2015/07/10/kamerbrief-over-convenantpreventie-gehoorschade-muzieksector.

[34] M. Kok "Sound Level Measurements and Control at Large Dance Events," presented at the AES 58th International Conference: Music Induced Hearing Disorders (2015 June), conference paper 4-2.

[35] J. Navne "Sound Level Measurements Made Eazy: 10 Eazy-Intuitive SPL Monitoring for Live Sound Events presented at the AES 58th International Conference: Music Induced Hearing Disorders (2015 June), conference paper 4-3.

[36] C. Borg "An Investigation of the Sound Pressure Level at the Roskilde Festival," presented at the AES 58th International Conference: Music Induced Hearing Disorders (2015 June), conference paper 4-4.

[37] E. Skovenborg "Loudness Range (LRA)—Design and Evaluation," presented at the 132nd Convention of the Audio Engineering Society (2012 Apr.), convention paper 8616.

[38] T. McMinn, “'A-Weighting': Is it the Metric You Think it Is," Proc. Accost., pp. 17-20 (2013 Nov.).

[39] Jr. R. Pierre and D. J. Maguire "The Impact of AWeighting Sound Pressure Level Measurements during the Evaluation of Noise Exposure," Conference NOISE-CON (2004).

[40] J. Mulder "Amplified Music and Sound Level Management; A Multi Stakeholder Perspective," presented at the AES 58th International Conference: Music Induced Hearing Disorders (2015 June), conference paper 4-1.

[41] J. Mulder Making Things Louder: Amplified Music and Multimodality, FASS (2013); Available from: http://hdl.handle.net/10453/21903. 


\section{THE AUTHOR}

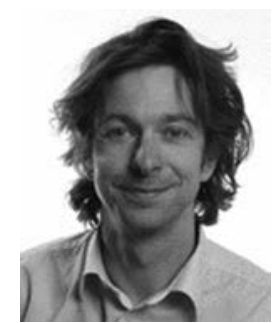

Johannes Mulder

Jos Mulder is a passionate sound technologist, researcher, and educator. Music is the gist in his broad education and professional experience. Initially trained as a recording engineer he specialized in live sound, working in the industry for 15 years. Additionally he developed an interest in the wider organizational, socio-cultural, and historical aspects of the use of electronic amplification in the performance arts. A Master's degree in arts management and administration (2008) and a Ph.D. (2013) looked at bridging the gap between technology, its creative use and broader discussions of the performance arts and society. Currently he is a tenured academic at Murdoch University in Perth teaching technological and sound studies subjects. 